\title{
Design and Implementation of Line Status Monitoring and Automatic Configuration Software for Aerospace Communication IP Network
}

\author{
Fei Yan, Chunyan Xie, Zhaoyang Cao, Zhen Liu and Leilei Li \\ China Satellite Maritime Tracking and Control Department, Jiangyin 214431, China
}

Keywords: aerospace communication IP network, design, condition monitoring.

\begin{abstract}
In view of the common network monitoring and maintenance methods can not meet the needs of aerospace communication IP network management, this paper based on aerospace communication IP network characteristics, research and development of auxiliary monitoring system to achieve the important line status monitoring and a variety of commonly used configuration changes, Improve the accuracy, can greatly improve the fault detection, testing, generation and other work efficiency.
\end{abstract}

\section{Introduction}

Aerospace communication IP network is mainly used in real-time data, voice, image service integrated transmission and exchange, requiring business to reliable, uninterrupted transmission, dynamic master network equipment and line status is important.

\section{Present Situation and Demand Analysis of Aerospace Communication IP Network}

The existing aerospace communication IP network status monitoring equipment is Huawei's U2000 network management system, the function is more powerful, but there are also a series of problems. Monitoring information is not detailed; especially the monitoring of external important line state there is a blind spot. The current monitoring system can not display the link status of the port. It can only display the physical connection status. If the intermediate node of the link fails, the existing network maintenance software can not be found. Job personnel through the manual one by one input command to configure changes, in particular, need to urgently on behalf of the situation, the manual operation takes a long time, the efficiency is not high, easy to mistake the problem is more obvious. In response to the above questions, put forward a few needs:

(A). Network critical link monitoring requirements

The requirements of the communication quality in the task are extremely strict, and it is very important to monitor the real-time status of the link in the network. The important link is configured by the static route so that the conventional network management software can not be monitored. Do the interface status monitoring, the software will focus on solving the conventional network management software is not easy to monitor the important link state.

(B). Routing fast switching requirements

The configuration of the route switchover is changed manually by the manual. Because the configuration item is long and similar, this method not only slows the switch speed, but also makes mistakes. The project is configured by the operator to determine the corresponding routing strategy. Background automatically modified to maximize the avoidance of errors caused by manual errors.

(C). Important direction of the main line fast road switching needs

In the main line failure will switch to a standby link, switching time directly affect the link interruption time, so this work on the high requirements of the previous manual mode of operation because of the configuration changes more, the operation is not fast enough, the software The same to achieve automatic changes, switching speed and no error.

(D). Save the operational record requirements 
Software in a variety of operations and important link problems occur, need to be recorded in order to facilitate the late network maintenance and troubleshooting, such as the emergence of network routing error, you can check the operating record database to see if the staff misuse.

\section{Software structure design}

According to the needs of the software, we will be divided into three main software, that is, the protocol layer, management, application layer. As shown in Figure 1 system architecture diagram.

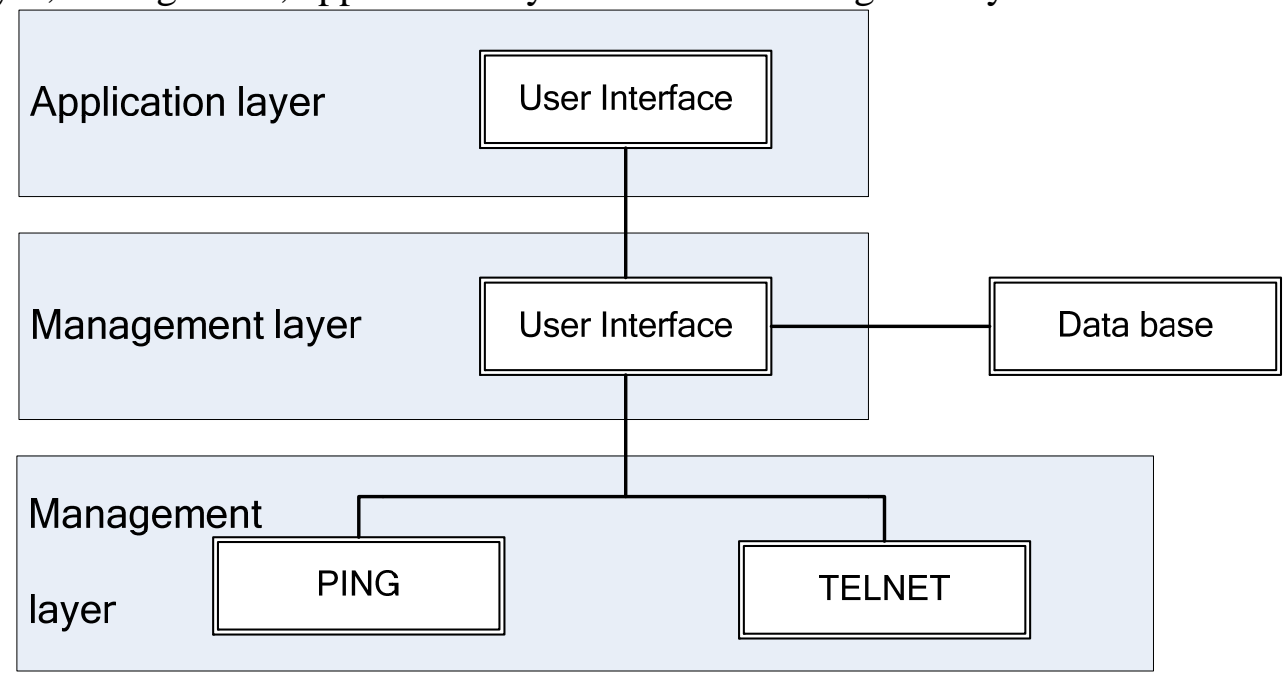

Figure 1. System architecture diagram

As the basis of the software, the main agreement to achieve the following:

(A). PING

As the most commonly used network management tool, this tool is simple. You can directly call the windows operating system PING tool to achieve the remote device network status measurement. However, due to the unsightly interface of the method and the poor statistical capability of the link continuous on-off test, such as background operation, the real-time redirection output is more complicated.

So the final design of the use of $\mathrm{C}$ \# ping class, the use of socket customization of the ping, to maintain a continuous interval to continue to send icmp packets without waiting to receive the message, the relative call to the system to bring their own ping tool monitoring link more sensitive, Of the message to calculate the final packet loss, delay and network off the situation.

(B). TELNET

The software uses telnet to remote management of the device, the original design for the direct call telnet command, in order to achieve the relevant command input. In practice, it is found that telnet requires a second call to be used. At this time, it is more difficult to redirect the input and output. Therefore, the modification scheme uses the socket socket to communicate with the device directly to simulate the manual operation of the device.

\section{Software implementation}

The software uses C / S architecture design, the important link state and operation records through the database real-time preservation, in order to facilitate the operator to observe the state of the device; the software interface is more concise and intuitive, mainly by the three major functional modules.

\subsection{Important link monitoring module}

The important link monitoring module mainly realizes the real-time monitoring of each direction link, in the way of ping. In order to make the monitoring better, the module does not call the system to integrate the ping command, but through the Socket socket custom way to achieve the ping function, this method makes the reported message frequency control, and through the analysis of the message 
can be analyzed Network performance parameters. The actual effect shown in Figure 2 module effect diagram shows:

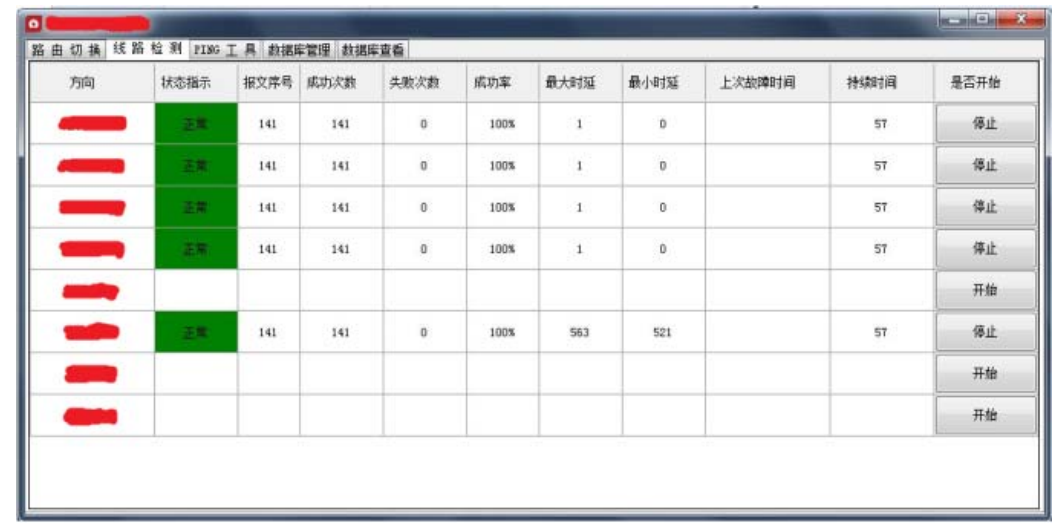

\subsection{Routing Fast Switching Module}

Figure 2. System architecture diagram

In the actual work of the system, TCP is the communication protocol, Ipv4 is used as the addressing scheme to establish the socket socket to establish the connection with the remote device and start to accept the data from the socket connection, and then accept the authentication request from the telnet server of the remote device After sending a predetermined user name and password, the final system will be the corresponding routing priority command sent to the connection Socket and from the Socket connection to accept data to complete the interaction between users and remote devices.

In the preparation of the routing fast switching module, the preparation of the class ScriptingTelnet, in the class ScriptingTelnet, the main realization of Connect, Disconnect, SendMessage, DoSend, Negotiate, OnRecievedData, WaitFor, Waitandsend, SessionLog, ClearSessionLog method. This article focuses on the five underlying methods of Negotiate, OnRecievedData, WaitFor, DoSend, Connect.

(A). Negotiate method is mainly negotiated with the Telnet server-side communication control code, the method we add a series of StringBuilder String type of decimal control code.

(B). DoSend method mainly to how to send data to the Telnet server side, the method we mainly use the Socket.send method and loop statements to send data.

(C). The OnRecievedData method mainly implements how to receive data from a Socket connection and decodes the data into English, which is stored in strWorkingData, strFullLog. In this method, we will decode the control code obtained from the Negotiate method and the data obtained from the Socker. Decode the results assigned to the strWorkingData, strFullLog these two String type variables.

(D). Waitfor method is mainly to achieve the Telnet client in the release of the next order before the server waiting for the implementation of a command on the feedback. In this method, we define the type long variable lngStart to record the time, define the type String variable Breaks to record the data, and use time to determine whether to send a timeout.

(E). Connect method to achieve the main socket socket assembly and the establishment of the connection. In this method, we first define the addressing mode as ipv4 in socket instance s, the socket type is stream, the communication protocol is TCP and then decide whether the connection is successful or not.

Other methods Disconnect is to close the Socket connection, SendMessage is called Dosend method to send the user input command, Waitandsend method is to call the Waitfor and Dosend methods to ensure that the user sends the data should not be network delay and other problems, the SessionLog method is to record and print from OnRecievedData get the data, ClearSessionLog method is to clear the data. 
Part of the code is as follows:

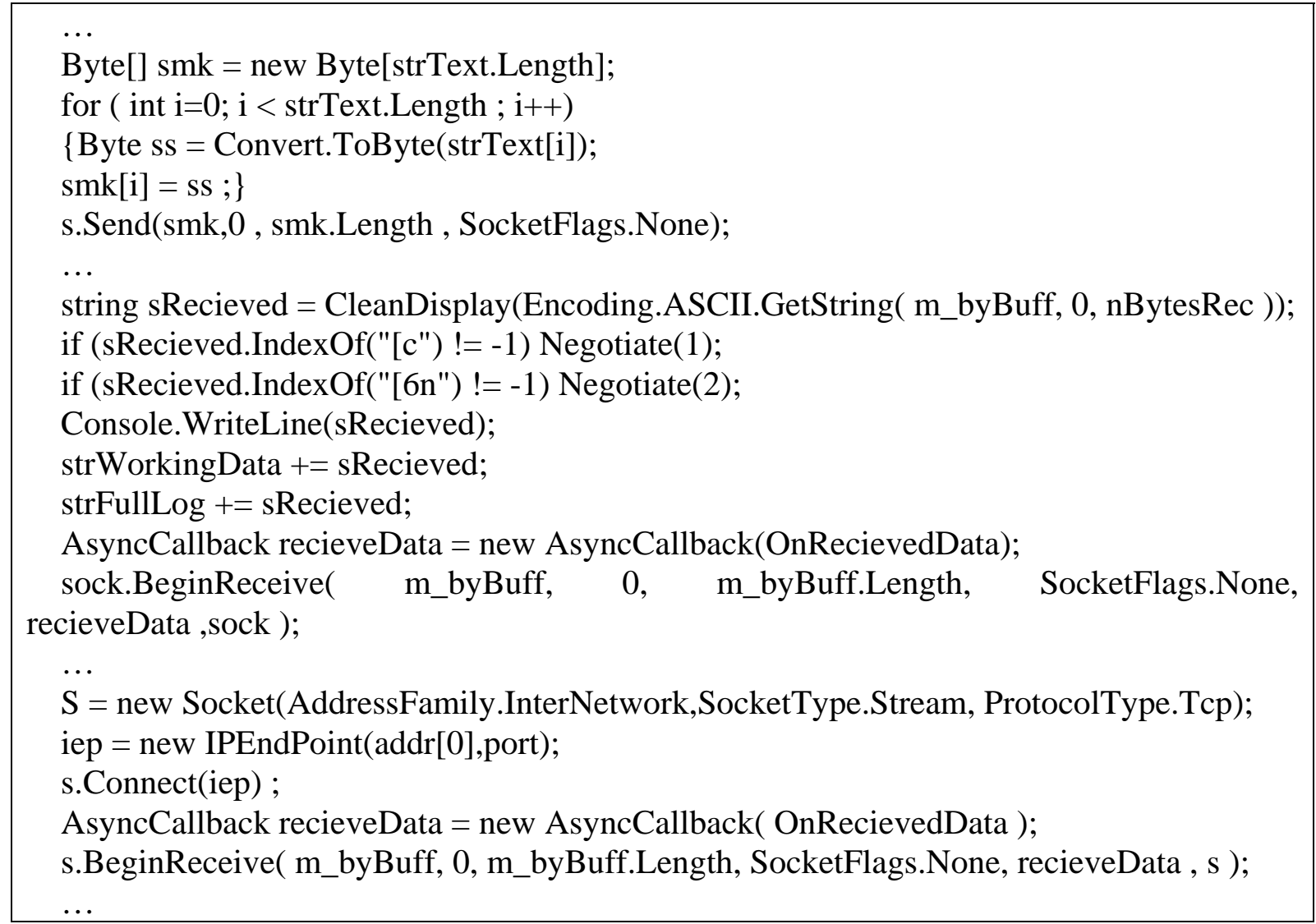

\section{Summary}

The development of the software will be the network of professional to the intelligent, unmanned network of professional first step forward, is an important part of the digital network professional. For how to automatically repair network failure, automatically determine the network failure, unattended network center will be the system more long-term goal. The software can fundamentally solve the problem of network fault monitoring found late, positioning difficult, and maintenance trouble and so on, greatly improve the efficiency of the daily work of network professionals, greatly reducing the workload of the post. The software can quickly help the technical staff to configure the network equipment, a great convenience to the technical staff of the network equipment configuration for the future development of network professionals to provide an important interface, but also for the network of professional automatic troubleshooting, automatic network Error, automatic network optimization provides an important technical basis.

\section{References}

[1] JIN Dong-li, MA Li-bo.Study on the Development Planning of Satellite Communication Network in China Aerospace Scientific Research [J] Satellite and Network, 2 010,9 6: 26-29.

[2] Li Kang, Chen Xuejun, Zhao Qianhong, Xu Jun, Shen Yu. Aerospace communication IP network multicast common fault solution [J]. Telemetry remote control, 2012,33 (2): 58-62.

[3] LU Shao-hua, LI Kang, LIN Xi-liang, ZHAO Qian-hong, ZHAO Jing-lei. Design and Implementation of Integrated Network Management System for Aerospace Experiment Communication Network [J]. Telemetry and Remote Control, 2015,36 (4): 71-76. 